\title{
Properties of Fuzzy Length on Fuzzy Set
}

\section{Jehad R. Kider, Jaafar Imran Mousa}

Department of Mathematics and Computer Applications, School of Applies Sciences, University of Technology, Bagdad, Iraq

Email: jehadkider@gmail.com

How to cite this paper: Kider, J.R. and Mousa, J.I. (2016) Properties of Fuzzy Length on Fuzzy Set. Open Access Library Journal, 3: e3068.

http://dx.doi.org/10.4236/oalib.1103068

Received: September 15, 2016

Accepted: November 13, 2016

Published: November 17, 2016

Copyright @ 2016 by authors and Open Access Library Inc.

This work is licensed under the Creative Commons Attribution International

License (CC BY 4.0).

http://creativecommons.org/licenses/by/4.0/

\section{Abstract}

The definition of fuzzy length space on fuzzy set in this research was introduced after the studies and discussion of many properties of this space were proved, and then an example to illustrate this notion was given. Also the definition of fuzzy convergence, fuzzy bounded fuzzy set, and fuzzy dense fuzzy set space was introduced, and then the definition of fuzzy continuous operator was introduced.

\section{Subject Areas}

Fuzzy Mathematics

\section{Keywords}

Fuzzy Length Space on Fuzzy Set, Fuzzy Convergence, Fuzzy Cauchy Sequence of Fuzzy Point, Fuzzy Bounded Fuzzy Set and Fuzzy Continuous Operator

\section{Introduction}

Zadeh in 1965 [1] introduced the theory of fuzzy sets. Many authors have introduced the notion of fuzzy norm in different ways [2]-[9]. Cheng and Mordeson in 1994 [10] defined fuzzy norm on a linear space whose associated fuzzy metric is of Kramosil and Mickalek type [11] as follows:

The order pair $(X, N)$ is said to be a fuzzy normed space if $X$ is a linear space and $N$ is a fuzzy set on $X \times[0, \infty)$ satisfying the following conditions for every $x, y \in X$ and $s, t \in[0, \infty)$.

(i) $N(x, 0)=0$, for all $x \in X$.

(ii) For all $t>0, N(x, t)=1$ if and only if $x=0$.

(iii) $N(\alpha x, t)=N\left(x, \frac{t}{|\alpha|}\right)$, for all $\alpha \neq 0$ and for all $t>0$.

(iv) For all $s, t>0, N(x+y, t+s) \geq N(x, t) \wedge N(y, s)$ where $a \wedge b=\min \{a, b\}$.

(v) $\lim _{t \rightarrow \infty} N(x, t)=1$. 
The definition of continuous $t$-norm was introduced by George and Veeramani in [12]. Bag and Samanta in [2] modified the definition of Cheng and Mordeson of fuzzy norm as follows:

The triple $(X, N, *)$ is said to be a fuzzy normed space if $X$ is a linear space, * is a continuous $t$-norm and $N$ is a fuzzy set on $X \times[0, \infty)$ satisfying the following conditions for every $x, y \in X$ and $s, t \in[0, \infty)$.

(i) $N(x, 0)=0$, for all $x \in X$.

(ii) For all $t>0, N(x, t)=1$ if and only if $x=0$.

(iii) $N(\alpha x, t)=N\left(x, \frac{t}{|\alpha|}\right)$ for all $\alpha \neq 0$.

(iv) For all $s, t>0, N(x, t) * N(y, s) \leq N(x+y, t+s)$.

(v) For $x \neq 0, N(x, \cdot):(0, \infty) \rightarrow[0,1]$ is continuous.

(vi) $\lim _{t \rightarrow \infty} N(x, t)=1$.

The definition of fuzzy length space is introduced in this research as a modification of the notion of fuzzy normed space due to Bag and Samanta. In Section 1, we recall basic concepts of fuzzy set and the definition of continuous $t$-norm. Then in Section 2 we define the fuzzy length space on fuzzy set after we give an example; then we prove that every ordinary norm induces a fuzzy length, and also the definition of fuzzy open fuzzy ball, fuzzy convergent sequence, fuzzy open fuzzy set, fuzzy Cauchy sequence, and fuzzy bounded fuzzy set is introduced. In Section 3, we prove other properties of fuzzy length space. Finally in Section 4, we define a fuzzy continuous operator between two fuzzy length spaces. Also we prove several properties for fuzzy continuous operator.

\section{Basic Concept about Fuzzy Set}

\section{Definition 2.1:}

Let $X$ be a classical set of object, called the universal set, whose generic elements are denoted by $x$. The membership in a classical subject $A$ of $X$ is often viewed as a characteritic function $\mu_{A}$ from $X$ onto $\{0,1\}$ such that $\mu_{A}=1$ if $x \in A$ and $\mu_{A}=0$ if $x \notin A,\{0,1\}$ is called a valuation set. If a valuation set is allowed to be real interval $[0,1]$ then $A$ is called a fuzzy set which is denoted in this case by $\tilde{A}$ and $\mu_{\tilde{A}}$ is the grade of membership of $x$ in $\tilde{A}$. Also, it is remarkable that the closer the value of $\mu_{\tilde{A}}(x)$ to 1 , the more belong to $\tilde{A}$. Clearly, $\tilde{A}$ is a subset of $X$ that has no sharp boundary. The fuzzy set $\tilde{A}$ is completely characterized by the set of pairs:

$$
\tilde{A}=\left\{\left(x, \mu_{\tilde{A}}(x)\right): x \in X, 0 \leq \mu_{\tilde{A}}(x) \leq 1\right\} \quad[1] .
$$

\section{Definition 2.2:}

Suppose that $\tilde{D}$ and $\tilde{B}$ are two fuzzy sets in $Y$. Then

(i) $\tilde{D} \subseteq \tilde{B} \Leftrightarrow \mu_{\tilde{D}}(y) \leq \mu_{\tilde{B}}(y) \forall y \in Y$.

(ii) $\tilde{D}=\tilde{B} \Leftrightarrow \mu_{\tilde{D}}(y)=\mu_{\tilde{B}}(y) \forall y \in Y$.

(iii) $\tilde{C}=\tilde{D} \cup \tilde{B} \Leftrightarrow \mu_{\tilde{C}}(y)=\mu_{\tilde{D}}(y) \vee \mu_{\tilde{B}}(y) \quad \forall y \in Y$.

(iv) $\tilde{E}=\tilde{A} \cap \tilde{B} \Leftrightarrow \mu_{\tilde{E}}(y)=\mu_{\tilde{D}}(y) \wedge \mu_{\tilde{B}}(y) \forall y \in Y$. 
(v) $\mu_{\tilde{D}^{c}}(x)=1-\mu_{\tilde{D}}(y) \forall y \in Y \quad[4]$.

\section{Definition 2.3:}

Suppose that $\tilde{D}$ and $\tilde{B}$ be two fuzzy sets in $V \neq \varnothing$ and $W \neq \varnothing$ respectively then $\tilde{D} \times \tilde{B}$ is a fuzzy set whose membership is defined by:

$$
\mu_{\tilde{D} \times \tilde{B}}(a, b)=\mu_{\tilde{D}}(a) \wedge \mu_{\tilde{B}}(b) \quad \forall(a, b) \in V \times W \quad[8] .
$$

\section{Definition 2.4:}

A fuzzy point $p$ in $Y$ is a fuzzy set with single element and is denoted by $x_{\alpha}$ or $(x, \alpha)$. Two fuzzy points $x_{\alpha}$ and $y_{\beta}$ are said to be different if and only if $x \neq y$ [11].

\section{Definition 2.5:}

Suppose that $d_{\beta}$ is a fuzzy point and $\tilde{D}$ is a fuzzy set in $Y$. then $d_{\beta}$ is said to belongs to $\tilde{D}$ which is written by $d_{\beta} \in \tilde{D} \Leftrightarrow$ if $\mu_{\tilde{D}}(x)>\beta \quad$ [2].

\section{Definition 2.6:}

Suppose that $h$ is a function from the set $V \neq \varnothing$ into the set $W \neq \varnothing$. Let $\tilde{D}$ be a fuzzy set in $W$ then $h^{-1}(\tilde{D})$ is a fuzzy set in $V$ its membership is:

$\mu_{h^{-1}(\tilde{D})}(d)=\left(\mu_{\tilde{D}} \circ h\right)(d)$ for all $d$ in $V$. Also when $\tilde{E}$ is a fuzzy set in $V$ then $h(\tilde{E})$ is a fuzzy set in $W$ its membership is given by: $\mu_{h(\tilde{E})}(y)=\vee\left\{\mu_{\tilde{E}}(d): d \in f^{-1}(y)\right\}$ when $f^{-1}(y) \neq \varnothing$ and $\mu_{h(\tilde{E})}(y)=0$ otherwise [5].

\section{Proposition 2.7:}

Suppose that $h: V \rightarrow W$ is a function. Then the image of the fuzzy point $d_{\beta}$ in $V$, is the fuzzy point $h\left(d_{\beta}\right)$ in W with $h\left(d_{\beta}\right)=(h(d), \beta)$ [11].

\section{Definition 2.8:}

A binary operation $*:[0,1]^{2} \rightarrow[0,1]$ is said to be $\mathrm{t}$-norm (or continuous triangular norm) if $\forall p, q, t, r \in[0,1]$ the conditions are satisfied:

(i) $p * q=p * q$.

(ii) $p * 1=p$.

(iii) $(p * q) * t=p *(q * t)$.

(iv) If $p \leq q$ and $t \leq r$ then $p * t \leq q * r$ [12].

\section{Examples 2.9:}

When $p * q=p \cdot q$ and $p * q=p \wedge q \quad \forall p, q \in[0,1]$ then $*$ is a continuous $t$ norm [10].

\section{Remark 2.10:}

$\forall p>q$, there is $\mathrm{t}$ such that $p * t \geq q$ and for every $r$, there is e such that $r * r \geq e$, where $p, q, t, r$ and e belongs to [0,1] [12].

\section{Definition 2.11:}

Let $\tilde{D}$ be a fuzzy set in $Z$ and let $\tau$ be a collection of all subset fuzzy set in $\tilde{D}$. then $(\tilde{D}, \tau)$ is called a fuzzy topological space on the fuzzy set $\tilde{D}$ if (i) $\tilde{D}, \phi \in \tau$.

(ii) $\bigcap_{i=1}^{n} \tilde{E}_{i} \in \tau$ for any $\tilde{E}_{i} \in \tau, i=1,2, \cdots, n$.

(ii) $\bigcup_{i=1}^{\infty} \tilde{E}_{i} \in \tau$, for $\left\{\tilde{E}_{i}: \tilde{E}_{i} \in \tau, i=1,2, \cdots, \infty\right\}[10]$.

\section{Proposition 2.12:}


Let $T: \tilde{A} \rightarrow \tilde{D}$ be an arbitrary operator and $\tilde{E} \subseteq \tilde{A}$ and $\tilde{C} \subseteq \tilde{D}$. Then $T(\tilde{E}) \subseteq \tilde{C}$ if and only if $\tilde{E} \subseteq T^{-1}(\tilde{C})$ [9].

\section{On Fuzzy Length Space}

First we introduce the main definition in this paper.

\section{Definition 3.1:}

Let $X$ be a linear space over field $\mathbb{K}$ and let $\tilde{A}$ be a fuzzy set in $X$. let $*$ be a $t$ norm and $\tilde{F}$ be a fuzzy set from $\tilde{A}$ to $[0,1]$ such that:

$\left(\mathrm{FL}_{1}\right) \tilde{F}\left(x_{\alpha}\right)>0$ for all $x_{\alpha} \in \tilde{A}$.

$\left(\mathrm{FL}_{2}\right) \quad \tilde{F}\left(x_{\alpha}\right)=1$ if and only if $x_{\alpha}=0$.

$\left(\mathrm{FL}_{3}\right) \quad \tilde{F}(c x, \alpha)=\tilde{F}\left(x, \frac{\alpha}{|c|}\right)$ where $0 \neq c \in \mathbb{K}$.

$\left(\mathrm{FL}_{4}\right) \quad \tilde{F}\left(x_{\alpha}+y_{\beta}\right) \geq \tilde{F}\left(x_{\alpha}\right) * \tilde{F}\left(y_{\beta}\right)$.

$\left(\mathrm{FL}_{5}\right) \quad \tilde{F}$ is a continuous fuzzy set for all $x_{\alpha}, y_{\beta} \in \tilde{A}$ and $\alpha, \beta \in[0,1]$.

Then the triple $(\tilde{A}, \tilde{F}, *)$ is called a fuzzy length space on the fuzzy set $\tilde{A}$.

\section{Definition 3.2:}

Suppose that $(\tilde{D}, \tilde{F}, *)$ is a fuzzy length space on the fuzzy set $\tilde{D}$ then $\tilde{F}$ is continuous fuzzy set if whenever $\left\{\left(x_{n}, \infty_{n}\right)\right\} \rightarrow x_{\alpha}$ in $\tilde{D}$ then $\tilde{F}\left\{\left(x_{n}, \infty_{n}\right)\right\} \rightarrow \tilde{F}\left(x_{\alpha}\right)$ that is $\lim _{n \rightarrow \infty} \tilde{F}\left[\left(x_{n}, \propto_{n}\right)\right]=\tilde{F}\left(x_{\alpha}\right)$.

\section{Proposition 3.3:}

Let $(Y,$.$) be a normed space, suppose that \tilde{D}$ be a fuzzy set in $Y$. Put $\left\|x_{\alpha}\right\|=\|x\|$. Then $(\tilde{D},$.$) , is a fuzzy normed space.$

\section{Proof:}

Let $x_{\alpha}, y_{\beta} \in \tilde{D}$ and $0 \neq c \in \mathbb{K}$ then

1) $\left\|x_{\alpha}\right\|>0$ for all $x_{\alpha} \in \tilde{D}$.

2) $\left\|x_{\alpha}\right\|=0 \Leftrightarrow\|x\|=0 \Leftrightarrow x=0 \Leftrightarrow x_{\alpha}=0$.

3) $\|(c x, \alpha)\|=\|c x\|=\|c\|\|x\|=\|c\|\left\|x_{\alpha}\right\|$.

4) $\left\|x_{\alpha}+y_{\beta}\right\|=\|x+y\| \leq\|x\|+\|y\|=\left\|x_{\alpha}\right\|+\left\|y_{\beta}\right\|$.

Hence $(\tilde{D},\|\|$.$) , is a fuzzy normed space.$

\section{Example 3.4:}

Suppose that $(Y,\|\cdot\|)$ is a normed space and assume that $\tilde{D}$ is a fuzzy set in $Y$. Put $p * q=p \cdot q$ for all $p, q \in[0,1]$. Define $\tilde{F}_{\|\| \|}\left(x_{\alpha}\right)=\frac{\alpha}{\alpha+\|x\|}$.

Then $\left(\tilde{D}, \tilde{F}_{\| . \mid}, *\right)$ is a fuzzy length space on the fuzzy set $\tilde{D}$, is called the fuzzy length induced by $\|$.$\| .$

Proof:

To prove $\left(\tilde{D}, \tilde{F}_{\| . \mid}, *\right)$ is a fuzzy length space on the fuzzy set $\tilde{D}$ we must prove the five conditions of Definition 3.1:

$\left(\mathrm{FL}_{1}\right)$ Since $\|x\|>0$ for all $x \in X$ so $\tilde{F}_{\|\cdot\|}\left(x_{\alpha}\right)>0$ for all $x \in \tilde{D}$, where $\alpha>0$.

$\left(\mathrm{FL}_{2}\right)$ It is clear that $\tilde{F}_{\|.\|}\left(x_{\alpha}\right)=1$ for each $\alpha>0$ if and only if $x=0$.

$\left(\mathrm{FL}_{3}\right)$ If $0 \neq c \in \mathbb{K}$ then for each $x \in X$, 


$$
\begin{aligned}
\tilde{F}_{\|\| \|}(c x, \alpha)=\frac{\alpha}{\alpha+\|c x\|} & =\frac{\alpha}{\alpha+\|c\|\|x\|}=\tilde{F}_{\|\| \|}\left(x, \frac{\alpha}{|c|}\right) \text { for each } \alpha>0 . \\
\tilde{F}_{\|\| \|}\left(x_{\alpha}, y_{\beta}\right) & =\tilde{F}_{\|\|\|\|}(x+y, \lambda)=\frac{\lambda}{\lambda+\|x+y\|} \geq \frac{\lambda}{\lambda+\|x\|+\|y\|} \geq \frac{\lambda}{\lambda+\|x\|} \cdot \frac{\lambda}{\lambda+\|y\|} \\
& >\frac{\alpha}{\alpha+\|x\|} \cdot \frac{\beta}{\beta+\|y\|}=\tilde{F}_{\|\cdot\|}\left(x_{\alpha}\right) * \tilde{F}_{\|\| \|}\left(y_{\beta}\right)
\end{aligned}
$$

each $x_{\alpha}, y_{\beta} \in \tilde{A}$, where $\lambda=\min \{\alpha, \beta\}$.

$\left(\mathrm{FL}_{5}\right) \quad \tilde{F}_{\|.\|}\left(x_{\alpha}\right)$ is continuous since $\|x\|$ is a continuous function.

Hence $\left(\tilde{D}, \tilde{F}_{\|\| . \mid} *\right)$ is a fuzzy length space on the fuzzy set $\tilde{D}$.

\section{Definition 3.5:}

Let $\tilde{A}$ be a fuzzy set in $X$, and assume that $(\tilde{A}, \tilde{F}, *)$ is a fuzzy length space on the fuzzy set $\tilde{A}$. Let $\tilde{B}\left(x_{\alpha}, r\right)=\left\{y_{\beta} \in \tilde{A}: \tilde{F}\left(y_{\beta}-x_{\alpha}\right)>(1-p)\right\}$. So $\tilde{B}\left(x_{\alpha}, p\right)$ is said to be a fuzzy open fuzzy ball of center $x_{\infty} \in \tilde{A}$ and radius $r$.

We omitted the proof of the next result since it is clear.

\section{Proposition 3.6:}

In the fuzzy length space $(\tilde{A}, \tilde{F}, *)$ on the fuzzy set $\tilde{A}$, Let $\tilde{B}\left(x_{\alpha}, r_{1}\right)$ and $\tilde{B}\left(x_{\alpha}, r_{2}\right)$ with $x_{\alpha} \in \tilde{A}$ and $r_{1}, r_{2} \in(0,1)$. Then either $\tilde{B}\left(x_{\alpha}, r_{1}\right) \subseteq \tilde{B}\left(x_{\alpha}, r_{2}\right)$ or $\tilde{B}\left(x_{\alpha}, r_{2}\right) \subseteq \tilde{B}\left(x_{\alpha}, r_{1}\right)$.

\section{Definition 3.7:}

The sequence $\left\{\left(x_{n}, \propto_{n}\right)\right\}$ in a fuzzy length space $(\tilde{A}, \tilde{F}, *)$ on the fuzzy set $\tilde{A}$ is fuzzy converges to a fuzzy point $x_{\alpha} \in \tilde{A}$ if for a given $\varepsilon, 0<\varepsilon<1$, then there exists a positive number $K$ such that $\tilde{F}\left[\left(x_{n}, \propto_{n}\right)-x_{\alpha}\right]>(1-\varepsilon) \forall n \geq K$.

\section{Definition 3.8:}

The sequence $\left\{\left(x_{n}, \propto_{n}\right)\right\}$ in a fuzzy length space $(\tilde{A}, \tilde{F}, *)$ on the fuzzy set $\tilde{A}$ is fuzzy converges to a fuzzy point $x_{\alpha} \in \tilde{A}$ if $\lim _{n \rightarrow \infty} \tilde{F}\left[\left(x_{n}, \propto_{n}\right)-x_{\alpha}\right]=1$.

\section{Theorem 3.9:}

The two Definitions 3.8 and 3.7 are equivalent.

\section{Proof:}

Let the sequence $\left\{\left(x_{n}, \propto_{n}\right)\right\}$ is fuzzy converges to a fuzzy point $x_{\alpha} \in \tilde{A}$, then for a given $0<\varepsilon<1$ then there is a number $K$ with $\tilde{F}\left[\left(x_{n}, \propto_{n}\right)-x_{\alpha}\right]>(1-r)$ for all $n \geq K$, and hence $1-\tilde{F}\left[\left(x_{n}, \infty_{n}\right)-x_{\alpha}\right]<r$. Therefore $\tilde{F}\left[\left(x_{n}, \infty_{n}\right)-x_{\alpha}\right] \rightarrow 1$ when $n \rightarrow \infty$.

To prove the converse, let $\tilde{F}\left[\left(x_{n}, \infty_{n}\right)-x_{\alpha}\right] \rightarrow 1$ when $n \rightarrow \infty$.

Hence when $0<\varepsilon<1$, there is $K$ such that $1-\tilde{F}\left[\left(x_{n}, \propto_{n}\right)-x_{\alpha}\right]<r \quad \forall n \geq K$.

So $\tilde{F}\left[\left(x_{n}, \propto_{n}\right)-x_{\alpha}\right]>(1-r) \forall n \geq K$. Therefore $\left\{\left(x_{n}, \propto_{n}\right)\right\} \rightarrow x_{\alpha}$ by Definition 3.7.

\section{Lemma 3.10:}

Suppose that $(\tilde{A}, \tilde{F}, *)$ is a fuzzy length space on the fuzzy set $\tilde{A}$. Then $\tilde{F}\left(x_{\alpha}-y_{\beta}\right)=\tilde{F}\left(y_{\beta}-x_{\alpha}\right)$ for any $x_{\alpha}, y_{\beta} \in \tilde{A}$.

Proof:

Let $x_{\alpha}, y_{\beta} \in \tilde{A}$, then $\tilde{F}\left(x_{\alpha}-y_{\beta}\right)=\tilde{F}((x-y), t)$ where $t=\alpha \wedge \beta$ 


$$
=\tilde{F}(-1(y-x), t)=\tilde{F}\left((y-x), \frac{t}{|-1|}\right)=\tilde{F}((y-x), t)=\tilde{F}\left(y_{\beta}-x_{\alpha}\right) .
$$

\section{Lemma 3.11:}

If $(\tilde{A}, \tilde{F}, *)$ is a fuzzy length space. Then,

(a) The operator $\left(x_{\alpha}, y_{\beta}\right) \rightarrow x_{\alpha}+y_{\beta}$ is continuous.

(b) The operator $\left(r, x_{\alpha}\right) \rightarrow r x_{\alpha}$ is continuous.

Proof:

If $\left\{\left(x_{n}, \propto_{n}\right)\right\} \rightarrow x_{\alpha}$ and $\left\{\left(y_{n}, \beta_{n}\right)\right\} \rightarrow y_{\alpha}$ as $n \rightarrow \infty$ then

$$
\begin{aligned}
& \tilde{F}\left(\left[\left(x_{n}, \infty_{n}\right)+y_{n}, \beta_{n}\right]-\left(x_{\alpha}+y_{\beta}\right)\right) \\
& \geq \tilde{F}\left[\left(x_{n}, \propto_{n}\right)-x_{\alpha}\right] * \tilde{F}\left[\left(y_{n}, \beta_{n}\right)-y_{\beta}\right]=1 * 1=1
\end{aligned}
$$

Hence the operator addition is continuous function.

Now if $\left\{\left(x_{n}, \propto_{n}\right)\right\} \rightarrow x_{\alpha}$, and $r_{n} \rightarrow r$ and $r_{n} \neq 0$ then

$$
\begin{aligned}
& \lim _{n \rightarrow \infty} \tilde{F}\left[r_{n}\left(x_{n}, \propto_{n}\right)-r x_{\alpha}\right] \\
& =\lim _{n \rightarrow \infty} \tilde{F}\left[\left(r_{n} x_{n}, \propto_{n}\right)-r x_{\alpha}\right] \\
& =\lim _{n \rightarrow \infty} \tilde{F}\left[\left(r_{n} x_{n}, \propto_{n}\right)-r_{n} x_{\alpha}+r_{n} x_{\alpha}-r x_{\alpha}\right] \\
& =\lim _{n \rightarrow \infty} \tilde{F}\left[r_{n}\left(x_{n}-x_{\alpha}, \propto_{n}\right)+x_{\alpha}\left(r_{n}-r\right)\right] \\
& \geq \lim _{n \rightarrow \infty} \tilde{F}\left[x_{n}-x_{\alpha}, \frac{\propto_{n}}{\left|r_{n}\right|}\right] * \lim _{n \rightarrow \infty} \tilde{F}\left[x, \frac{\alpha}{\left|r_{n}-r\right|}\right] \geq 1 * 1=1
\end{aligned}
$$

And this proves (b).

\section{Definition 3.12:}

Suppose that $(\tilde{A}, \tilde{F}, *)$ is a fuzzy length space and $\tilde{D} \subseteq \tilde{A}$ then $\tilde{D}$ is called fuzzy open if for every $y_{\beta} \in \tilde{D}$ there is $\tilde{B}\left(y_{\beta}, q\right) \subseteq \tilde{D}$. A subset $\tilde{E} \subseteq \tilde{A}$ is called fuzzy closed if $\tilde{E}^{c}=\tilde{A}-\tilde{E}$ is fuzzy open.

The proof of the following theorem is easy and so is omitted.

\section{Theorem 3.13:}

Any $\tilde{B}\left(y_{\beta}, q\right)$ in a fuzzy length space $(\tilde{A}, \tilde{F}, *)$ is a fuzzy open.

\section{Definition 3.14:}

Suppose that $(\tilde{A}, \tilde{F}, *)$ is a fuzzy length space, and assume that $\tilde{D} \subseteq \tilde{A}$. Then the fuzzy closure of $\tilde{D}$ is denoted by $\overline{\tilde{D}}$ or $F C(\tilde{D})$ and is defined by $\overline{\overline{\tilde{D}}}$ is the smallest fuzzy closed fuzzy set that contains $\tilde{D}$.

\section{Definition 3.15:}

Suppose that $(\tilde{A}, \tilde{F}, *)$ is a fuzzy length space, and assume that $\tilde{D} \subseteq \tilde{A}$. Then $\tilde{D}$ is said to be fuzzy dense in $\tilde{A}$ if $\tilde{D}=\tilde{A}$ or $F C(\tilde{D})=\tilde{A}$.

Lemma 3.16:

Suppose that $(\tilde{A}, \tilde{F}, *)$ is a fuzzy length space, and assume that $\tilde{D} \subseteq \tilde{A}$, Then $d_{\alpha} \in \tilde{\tilde{D}}$ if and only if we can find $\left\{\left(d_{n}, \propto_{n}\right)\right\}$ in $\tilde{D}$ such that $\left(d_{n}, \propto_{n}\right) \rightarrow d_{\alpha}$.

\section{Proof:}

Let $d_{\alpha} \in \overline{\tilde{D}}$, if $d_{\alpha} \in \tilde{D}$ then we take the sequence of fuzzy points of the type $\left(d_{\alpha}, d_{\alpha}, \cdots, d_{\alpha}\right)$. If $d_{\alpha} \notin \tilde{D}$, we construct the sequence of fuzzy points $\left(d_{n}, \propto_{n}\right) \in \tilde{D}$ as 
follows: $\tilde{F}\left[\left(d_{n}, \propto_{n}\right)-d_{\alpha}\right]>\left(1-\frac{1}{n}\right)$ for each $n \in \mathbb{N}$.

The fuzzy ball $\tilde{B}\left(d_{\alpha}, \frac{1}{n}\right)$ contains $\left(d_{n}, \propto_{n}\right) \in \tilde{D}$ and $\left(d_{n}, \propto_{n}\right) \rightarrow d_{\alpha}$ since $\lim _{n \rightarrow \infty} \tilde{F}\left[\left(d_{n}, \propto_{n}\right)-d_{\alpha}\right]=1$.

Conversely assume that $\left(d_{n}, \propto_{n}\right) \in \tilde{D}$ and $\left(d_{n}, \propto_{n}\right) \rightarrow d_{\alpha}$ then $d_{\alpha} \in \tilde{D}$ or the fuzzy open fuzzy ball of $d_{\alpha}$ contains $\left(d_{n}, \propto_{n}\right)$ with $\left(d_{n}, \propto_{n}\right) \neq d_{\alpha}$, so $d_{\alpha}$ is a fuzzy limit fuzzy point of $\tilde{D}$. Hence $d_{\alpha} \in \overline{\tilde{D}}$.

\section{Other Properties of Fuzzy Length on Fuzzy Set}

\section{Theorem 4.1:}

Suppose that $(\tilde{A}, \tilde{F}, *)$ is a fuzzy length space and let that $\tilde{D} \subseteq \tilde{A}$, then $\tilde{D}$ is fuzzy dense in $\tilde{A}$ if and only if for any $a_{\alpha} \in \tilde{A}$ we can find $d_{\beta} \in \tilde{D}$ with $\tilde{F}\left[a_{\alpha}-d_{\beta}\right] \geq(1-\varepsilon)$ for some $0<\varepsilon<1$.

Proof:

Let $\tilde{D}$ be a fuzzy dense in $\tilde{A}$, and $a_{\alpha} \in \tilde{A}$ so $a_{\alpha} \in \overline{\tilde{D}}$ then using 3.16 we can find $\left\{\left(d_{n}, \propto_{n}\right)\right\} \in \tilde{D} \quad$ with $\quad \tilde{F}\left[\left(d_{n}, \propto_{n}\right)-a_{\alpha}\right]>(1-\varepsilon) \quad$ for $\quad$ all $n \geq K$. Take $d_{\beta}=\left(d_{k}, \propto_{k}\right), \tilde{F}\left[d_{\beta}-a_{\alpha}\right]>(1-\varepsilon)$.

To prove the converse, we must prove that $\tilde{A} \subseteq \overline{\tilde{D}}$. Let $a_{\alpha} \in \tilde{A}$ then there is $\left(d_{j}, \propto_{j}\right) \in \tilde{D} \quad$ such that $\tilde{F}\left[\left(d_{j}, \infty_{j}\right)-a_{\alpha}\right]>\left(1-\frac{1}{j}\right)$, where $j \in \mathbb{N}$. Now take $0<\varepsilon<1$ such that $\frac{1}{j}<\varepsilon$ for each $j \geq K$. Hence we have a sequence of fuzzy points $\left\{\left(d_{j}, \propto_{j}\right)\right\} \in \tilde{D}$ such that $\tilde{F}\left[\left(d_{j}, \propto_{j}\right)-a_{\alpha}\right]>\left(1-\frac{1}{j}\right)>(1-\varepsilon)$ for all $j \geq K$ that is $\left(d_{j}, \propto_{j}\right) \rightarrow a_{\alpha}$ so $a_{\alpha} \in \overline{\tilde{D}}$.

\section{Definition 4.2:}

Suppose that $(\tilde{A}, \tilde{F}, *)$ is a fuzzy length space. A sequence of fuzzy points $\left\{\left(x_{n}, \propto_{n}\right)\right\}$ is said to be a fuzzy Cauchy if for any given $\varepsilon, 0<\varepsilon<1$, there is a positive number $K$ such that $\tilde{F}\left[\left(x_{n}, \propto_{n}\right)-\left(x_{m}, \propto_{m}\right)\right]>(1-\varepsilon)$ for all $n, m \geq K$.

Theorem 4.3:

If $\left\{\left(x_{n}, \propto_{n}\right)\right\}$ is a sequence in a fuzzy length space $(\tilde{A}, \tilde{F}, *)$ with $\left(x_{n}, \propto_{n}\right) \rightarrow x_{\alpha}$ then $\left\{\left(x_{n}, \propto_{n}\right)\right\}$ is fuzzy Cauchy.

\section{Proof:}

If $\left\{\left(x_{n}, \propto_{n}\right)\right\}$ is a sequence in a fuzzy length space $(\tilde{A}, \tilde{F}, *)$ with $\left(x_{n}, \propto_{n}\right) \rightarrow x_{\alpha}$. So for any $\varepsilon, 0<\varepsilon<1$ there is an integer $K$ such that $\tilde{F}\left[\left(x_{n}, \infty_{n}\right)-x_{\alpha}\right]>(1-\varepsilon)$ for all $n \geq K$. Now by Remark 2.10 there is $(1-r) \in(0,1)$ such that $(1-\varepsilon) *(1-\varepsilon)>(1-r)$. Now for each $m, j \geq K$, we have

$$
\begin{aligned}
\tilde{F}\left[\left(x_{m}, \propto_{m}\right)-\left(x_{j}, \propto_{j}\right)\right] & \geq \tilde{F}\left[\left(x_{m}, \propto_{m}\right)-x_{\alpha}\right] * \tilde{F}\left[x_{\alpha}-\left(x_{j}, \propto_{j}\right)\right] \\
& \geq(1-\varepsilon) *(1-\varepsilon)>(1-r)
\end{aligned}
$$


Hence $\left\{\left(x_{n}, \infty_{n}\right)\right\}$ is fuzzy Cauchy.

\section{Definition 4.4:}

Suppose that $\left\{\left(x_{n}, \propto_{n}\right)\right\}$ is a sequence in a fuzzy length space $(\tilde{A}, \tilde{F}, *)$ let $\left(n_{k}\right)$ be a sequence of positive numbers such that $n_{1}<n_{2}<n_{3}<\cdots<n_{k}<\cdots$, then the sequence $\left\{\left(x_{n_{k}}, \infty_{n_{k}}\right)\right\}$ of fuzzy points is said to be a subsequence of $\left\{\left(x_{n}, \infty_{n}\right)\right\}$.

\section{Theorem 4.5:}

Suppose that $\left\{\left(x_{n}, \infty_{n}\right)\right\}$ is a sequence in a fuzzy length space $(\tilde{A}, \tilde{F}, *)$, if it is fuzzy converges to $x_{\alpha} \in \tilde{A}$ then every subsequence $\left\{\left(x_{n k}, \propto_{n k}\right)\right\}$ of $\left\{\left(x_{n}, \propto_{n}\right)\right\}$ is fuzzy converges to $x_{\alpha}$.

\section{Proof:}

Let $\left\{\left(x_{n}, \propto_{n}\right)\right\}$ be a sequence of fuzzy points in $(\tilde{A}, \tilde{F}, *)$ fuzzy converges to $x_{\alpha}$ then $\lim _{n \rightarrow \infty} \tilde{F}\left[\left(x_{n}, \infty_{n}\right)-x_{\alpha}\right]=1$. But $\left\{\left(x_{n}, \infty_{n}\right)\right\}$ is fuzzy Cauchy by Theorem (4.3) hence, $\lim \tilde{F}\left[\left(x_{n}, \infty_{n}\right)-\left(x_{m}, \infty_{m}\right)\right]=1$ as $n \rightarrow \infty$ and $m \rightarrow \infty$. Now

$$
\tilde{F}\left[\left(x_{n k}, \propto_{n k}\right)-x_{\alpha}\right] \geq \tilde{F}\left[\left(x_{n k}, \propto_{n k}\right)-\left(x_{n}, \propto_{n}\right)\right] * \tilde{F}\left[\left(x_{n}, \propto_{n}\right)-x_{\alpha}\right] .
$$

Taking the limit to both sides as $n \rightarrow \infty$ we get

$$
\begin{aligned}
& \lim _{n \rightarrow \infty} \tilde{F}\left[\left(x_{n k}, \infty_{n k}\right)-x_{\alpha}\right] \\
& \geq \lim _{n \rightarrow \infty} \tilde{F}\left[\left(x_{n k}, \propto_{n k}\right)-\left(x_{n}, \propto_{n}\right)\right] * \lim _{n \rightarrow \infty} \tilde{F}\left[\left(x_{n}, \propto_{n}\right)-x_{\alpha}\right] \\
& \geq 1 * 1=1
\end{aligned}
$$

Hence, $\left\{\left(x_{n k}, \infty_{n k}\right)\right\}$ fuzzy converges to $x_{\alpha}$, since $\left\{\left(x_{n k}, \infty_{n k}\right)\right\}$ was an arbitrary subsequence of $\left\{\left(x_{n}, \infty_{n}\right)\right\}$. Therefore all subsequence $\left\{\left(x_{n k}, \infty_{n k}\right)\right\}$ of $\left\{\left(x_{n}, \infty_{n}\right)\right\}$ is fuzzy converges to $x_{\alpha}$.

\section{Proposition 4.6:}

Let $\left\{\left(x_{n}, \infty_{n}\right)\right\}$ be a fuzzy Cauchy sequence in a fuzzy length space $(\tilde{A}, \tilde{F}, *)$ contains a $\left\{\left(x_{n_{k}}, \propto_{n_{k}}\right)\right\}$ such that $\left\{\left(x_{n_{k}}, \propto_{n_{k}}\right)\right\} \rightarrow x_{\alpha}$, then $\left\{\left(x_{n}, \propto_{n}\right)\right\}$ fuzzy converges to $x_{\alpha}$.

\section{Proof:}

Assume that $\left\{\left(x_{n}, \infty_{n}\right)\right\}$ is a fuzzy Cauchy sequence in $(\tilde{A}, \tilde{F}, *)$. So for all $\varepsilon$, $0<\varepsilon<1$ there is an integer $K$ such that $\tilde{F}\left[\left(x_{m}, \propto_{m}\right)-\left(x_{j}, \propto_{j}\right)\right]>(1-\varepsilon)$ whenever $m, j \geq K$. Let $\left\{\left(x_{n_{j}}, \propto_{n_{j}}\right)\right\}$ be a subsequence of $\left\{\left(x_{n}, \propto_{n}\right)\right\}$ and $\left(x_{n_{j}}, \propto_{n_{j}}\right) \rightarrow x_{\alpha}$. It follows that $\tilde{F}\left[\left(x_{n_{j}}, \infty_{n_{j}}\right)-x_{\alpha}\right] \rightarrow 1$ whenever $n_{j} \rightarrow \infty$. Since $\left(n_{j}\right)$ is increasing sequence of positive integers. Now

$$
\begin{aligned}
\tilde{F}\left[x_{\alpha}-\left(x_{n}, \propto_{n}\right)\right] & \geq \tilde{F}\left[x_{\alpha}-\left(x_{n_{m}}, \propto_{n_{m}}\right)\right] * \tilde{F}\left[\left(x_{n_{m}}, \propto_{n_{m}}\right)-\left(x_{n}, \propto_{n}\right)\right] \\
& >\tilde{F}\left[x_{\alpha}-\left(x_{n_{m}}, \propto_{n_{m}}\right)\right] *(1-\varepsilon)
\end{aligned}
$$

Letting $m \rightarrow \infty$, we have $\tilde{F}\left[x_{\alpha}-\left(x_{n}, \infty_{n}\right)\right] \geq 1 *(1-\varepsilon)=(1-\varepsilon)$.

Hence, the sequence $\left\{\left(x_{n}, \infty_{n}\right)\right\}$ it fuzzy converges to $x_{\alpha}$.

\section{Definition 4.7:}

Suppose that $(\tilde{A}, \tilde{F}, *)$ is a fuzzy length space and $\tilde{D} \subseteq \tilde{A}$. Then $\tilde{D}$ is said to be fuzzy bounded if we can find $q, 0<q<1 \quad 1$ such that, $\tilde{F}\left(x_{\alpha}\right)>(1-q) \forall x_{\alpha} \in \tilde{A}$. 


\section{Lemma 4.8:}

Assume that $(\tilde{A}, \tilde{F}, *)$ is a fuzzy length space. If a sequence $\left\{\left(x_{n}, \propto_{n}\right)\right\}$ with $\left(x_{n}, \propto_{n}\right) \rightarrow x_{\alpha}$ then it is fuzzy bounded and its fuzzy limit is unique.

Proof:

Let $\left(x_{n}, \propto_{n}\right) \rightarrow x_{\alpha}$, that is for a given $r>0$ then we can find $K$ with $\tilde{F}\left[\left(x_{n}, \propto_{n}\right)-x_{\alpha}\right]>(1-r)$ for all $n \geq K$.

Put $t=\min \left\{\tilde{F}\left[\left(x_{1}, \propto_{1}\right)-x_{\alpha}\right], \tilde{F}\left[\left(x_{2}, \propto_{2}\right)-x_{\alpha}\right], \cdots, \tilde{F}\left[\left(x_{K}, \propto_{K}\right)-x_{\alpha}\right]\right\}$.

Using Remark 2.10 we can find $0<\varepsilon<1$ with $t *(1-r)>(1-\varepsilon)$. Now for $n \geq K$

$$
\begin{aligned}
\tilde{F}\left[\left(x_{n}, \propto_{n}\right)-x_{\alpha}\right] & \geq \tilde{F}\left[\left(x_{n}, \propto_{n}\right)-\left(x_{K}, \propto_{K}\right)\right] * \tilde{F}\left[\left(x_{K}, \propto_{K}\right)-x_{\alpha}\right] \\
& \geq t *(1-r)>(1-\varepsilon) .
\end{aligned}
$$

Hence $\left(x_{n}, \propto_{n}\right)$ is fuzzy bounded in $(\tilde{A}, \tilde{F}, *)$.

Suppose that $\left(x_{n}, \propto_{n}\right) \rightarrow x_{\alpha}$ and $\left(x_{n}, \propto_{n}\right) \rightarrow y_{\beta}$. Therefore

$\lim _{n \rightarrow \infty} \tilde{F}\left[\left(x_{n}, \propto_{n}\right)-x_{\alpha}\right]=1$ and $\lim _{n \rightarrow \infty} \tilde{F}\left[\left(x_{n}, \propto_{n}\right)-y_{\beta}\right]=1$. Now

$\tilde{F}\left[x_{\alpha}-y_{\beta}\right] \geq \tilde{F}\left[x_{\alpha}-\left(x_{n}, \propto_{n}\right)\right] * \tilde{F}\left[\left(x_{n}, \propto_{n}\right)-y_{\beta}\right]$. By taking the limit to both sides, as $n \rightarrow \infty, \tilde{F}\left[x_{\alpha}-y_{\beta}\right] \geq 1 * 1=1$. So $\tilde{F}\left[x_{\alpha}-y_{\beta}\right]=1$, hence $x_{\alpha}=y_{\beta}$.

\section{Definition 4.9:}

Suppose that $(\tilde{A}, \tilde{F}, *)$ is a fuzzy length space on the fuzzy set $\tilde{A}$. Then $\tilde{B}\left[x_{\alpha}, q\right]=\left\{y_{\beta} \in \tilde{A}: \tilde{F}\left[x_{\alpha}-y_{\beta}\right] \geq(1-q)\right\}$ is said to be a fuzzy closed fuzzy ball with center $x_{\alpha} \in \tilde{A}$ and radius $q, 0<q<1$.

The proof of the following lemma is clear and hence is omitted.

\section{Lemma 4.10:}

Any $\tilde{B}\left[x_{\alpha}, q\right]$ in a fuzzy length space $(\tilde{A}, \tilde{F}, *)$ is a fuzzy closed fuzzy set.

\section{Theorem 4.11:}

A fuzzy length space $(\tilde{A}, \tilde{F}, *)$ is a fuzzy topological space.

\section{Proof:}

Suppose that $(\tilde{A}, \tilde{F}, *)$ is a fuzzy length space. Put $\tau_{\tilde{F}}=\left\{\tilde{D} \subseteq \tilde{A}: x_{\alpha} \in \tilde{D}\right\}$ if and only if there is $0<r<1$ such that $\tilde{B}\left(x_{\alpha}, r\right) \in \tilde{D}$. We prove that $\tau_{\tilde{F}}$ is a fuzzy topology on $\tilde{A}$.

(i) Clear that $\phi$ and $\tilde{A} \in \tau_{\tilde{F}}$.

(ii) let $\tilde{D}_{1}, \tilde{D}_{2}, \cdots, \tilde{D}_{n} \in \tau_{\tilde{F}}$ and put $\tilde{V}=\bigcap_{j=1}^{n} \tilde{D}_{j}$. We will show that $\tilde{V} \in \tau_{\tilde{F}}$, let $a_{\alpha} \in \tilde{V}$ then $a_{\alpha} \in \tilde{D}_{j}$ for each $1 \leq j \leq n$. Hence there is $0 \leq q_{j} \leq 1$ such that $\tilde{B}\left(a_{\alpha}, q_{j}\right) \subseteq \tilde{D}_{i}$. Put $q=\min \left\{q_{j}: 1 \leq j \leq n\right\}$ so $q \leq q_{j}$ for all $1 \leq j \leq n$, this implies that $(1-q) \geq\left(1-q_{j}\right)$ for all $1 \leq j \leq n$. Therefore, $\tilde{B}\left(a_{\alpha}, q_{j}\right) \subseteq \bigcap_{j=1}^{n} \tilde{D}_{j}=\tilde{V}$, thus $\tilde{V} \in \tau_{\tilde{F}}$.

(iii) Let $\left\{\tilde{D}_{j}: j \in I\right\} \in \tau_{\tilde{F}}$. put $\tilde{U}=\bigcup_{j \in I} \tilde{D}_{j}$. We will show that $\tilde{U} \in \tau_{\tilde{F}}$. Let $b_{\beta} \in \tilde{U}$, then $b_{\beta} \in \tilde{D}_{j}$ for some $j \in I$, since $\tilde{D}_{j} \in \tau_{\tilde{F}}$ then there exists $0<q<1$ such that $\tilde{B}\left(b_{\beta}, p\right) \subseteq \tilde{D}_{j}$, Hence $\tilde{B}\left(b_{\beta}, p\right) \subseteq \tilde{D}_{j} \subset \bigcup_{j \in I} \tilde{D}_{j}=\tilde{U}$. This prove that $\tilde{U} \in \tau_{\tilde{F}}$. Hence, $(\tilde{A}, \tilde{F}, *)$ is a fuzzy topological space. $\tau_{\tilde{F}}$ is called the fuzzy topology induced by $\tilde{F}$.

\section{Definition 4.12:}

A fuzzy length space $(\tilde{A}, \tilde{F}, *)$ is said to be a fuzzy Hausdorff space if for any $x_{\alpha}, y_{\beta} \in \tilde{A}$ such that $x_{\alpha} \neq y_{\beta}$ we can find $\tilde{B}\left(x_{\alpha}, r\right)$ and $\tilde{B}\left(y_{\beta}, t\right)$ for some $0<r<1$ 
and $0<t<1$ such that $\tilde{B}\left(x_{\alpha}, r\right) \cap \tilde{B}\left(y_{\beta}, t\right)=\varnothing$.

\section{Theorem 4.13:}

Every fuzzy length space $(\tilde{A}, \tilde{F}, *)$ is a fuzzy Hausdorff space.

\section{Proof:}

Assume that $(\tilde{A}, \tilde{F}, *)$ is a fuzzy length space and let $x_{\alpha}, \exists_{\beta} \in \tilde{A}$ with $x_{\alpha} \neq y_{\beta}$.

Put $\tilde{F}\left[x_{\alpha}-y_{\beta}\right]=p$, for some $0<p<1$. Then for each $s, 0<s<1$, we can find $q$ such that $q * q \geq s$ by Remark 2.10. Now consider the two fuzzy open fuzzy balls $\tilde{B}\left(x_{\alpha}, 1-q\right)$ and $\tilde{B}\left(y_{\beta}, 1-q\right)$. Then $\tilde{B}\left(x_{\alpha}, 1-q\right) \cap \tilde{B}\left(y_{\beta}, 1-q\right)=\varnothing$ Since if there exists $z_{\delta} \in \tilde{B}\left(x_{\alpha}, 1-q\right) \cap \tilde{B}\left(y_{\beta}, 1-q\right)$. Then

$$
p=\tilde{F}\left[x_{\alpha}-y_{\beta}\right] \geq \tilde{F}\left[x_{\alpha}-z_{\delta}\right] * \tilde{F}\left[z_{\delta}-y_{\beta}\right] \geq q * q \geq s>p
$$

is a contradiction, hence $(\tilde{A}, \tilde{F}, *)$ is a fuzzy Hausdorff space.

\section{Fuzzy Continuous Operators on Fuzzy Length Spaces}

In this section, we will suppose $\tilde{A}$ is fuzzy set of $X$ and $\tilde{D}$ be fuzzy set of $Y$ where $X$ and $Y$ are vector space.

\section{Definition 5.1:}

Let $\left(\tilde{A}, \tilde{F}_{\tilde{A}}, *\right)$ and $\left(\tilde{D}, \tilde{F}_{\tilde{D}}, *\right)$ be two fuzzy length space on fuzzy set $\tilde{A}$ and $\tilde{D}$ respectively, let $\tilde{E} \subseteq \tilde{A}$ then The operator $T: \tilde{E} \rightarrow \tilde{D}$ is said to be fuzzy continuous at $a_{\alpha} \in \tilde{E}$, if for every $0<\varepsilon<1$ there exist $0<\delta<1$ such that $\tilde{F}_{\tilde{D}}\left[T\left(x_{\beta}\right)-T\left(a_{\alpha}\right)\right]>(1-\varepsilon)$ whenever $x_{\beta} \in \tilde{E}$ satisfying $\tilde{F}_{\tilde{A}}\left(x_{\beta}-a_{\alpha}\right)>(1-\delta)$. If $T$ is fuzzy continuous at every fuzzy point of $\tilde{E}$, then $T$ it is said to be fuzzy continuous on $\tilde{E}$.

\section{Theorem 5.2:}

Let $\left(\tilde{A}, \tilde{F}_{\tilde{A}}, *\right)$ and $\left(\tilde{D}, \tilde{F}_{\tilde{D}}, *\right)$ be two fuzzy length space, let $\tilde{E} \subseteq \tilde{A}$. The operator $T: \tilde{E} \rightarrow \tilde{D}$ is fuzzy continuous at $a_{\alpha} \in \tilde{E}$ if and only if whenever a sequence of fuzzy points $\left\{\left(x_{n}, \alpha_{n}\right)\right\}$ in $\tilde{E}$ fuzzy converge to $a_{\alpha}$, then the sequence of fuzzy points $\left\{\left(T\left(x_{n}\right), \alpha_{n}\right)\right\}$ fuzzy converges to $T\left(a_{\alpha}\right)$.

\section{Proof:}

Suppose that the operator $T: \tilde{E} \rightarrow \tilde{D}$ is fuzzy continuous at $a_{\alpha} \in \tilde{E}$ and let $\left\{\left(x_{n}, \alpha_{n}\right)\right\}$ be a sequence in $\tilde{E}$ fuzzy converge to $a_{\alpha}$. Let $0<\varepsilon<1$ be given. By fuzzy continuity of $T$ at $a_{\alpha}$, then there exists $0<\delta<1$ such that whenever $x_{\beta} \in \tilde{E}$ and $\tilde{F}_{\tilde{A}}\left(x_{\beta}-a_{\alpha}\right)>(1-\delta)$, implies $\tilde{F}_{\tilde{D}}\left[T\left(x_{\beta}\right)-T\left(a_{\alpha}\right)\right]>(1-\varepsilon)$. Since $\left(x_{n}, \alpha_{n}\right) \rightarrow \alpha_{n}$ then we can find $K$ with $n \geq K$ such that $\tilde{F}_{\tilde{A}}\left[\left(x_{n}, \alpha_{n}\right)-a_{\alpha}\right]>(1-\delta)$. Therefore when $n \geq K$ implies $\tilde{F}_{\tilde{D}}\left[T\left(x_{n}, \alpha_{n}\right)-T\left(a_{\alpha}\right)\right]>(1-\varepsilon)$. Thus $T\left(x_{n}, \alpha_{n}\right) \rightarrow T\left(a_{\alpha}\right)$.

Conversely, assume that every sequence $\left\{\left(x_{n}, \alpha_{n}\right)\right\}$ in $\tilde{E}$ fuzzy converging to $a_{\alpha}$ has the property that $T\left(x_{n}, \alpha_{n}\right) \rightarrow T\left(a_{\alpha}\right)$. Suppose that $T$ is not fuzzy continuous at $a_{\alpha}$. Then there is $0<\varepsilon<1$ and for which no $\delta, 0<\delta<1$ can satisfy the requirement that $x_{\beta} \in \tilde{E}$ and $\tilde{F}_{\tilde{A}}\left[x_{\beta}-a_{\alpha}\right]>(1-\delta)$ implies $\tilde{F}_{\tilde{D}}\left[T\left(x_{\beta}\right)-T\left(a_{\alpha}\right)\right]>(1-\varepsilon)$. This means that for every $\delta, 0<\delta<1$ there exists $x_{\beta} \in \tilde{E}$ such that $\tilde{F}_{\tilde{A}}\left[x_{\beta}-a_{\alpha}\right]>(1-\delta)$ but $\tilde{F}_{\tilde{D}}\left[T\left(x_{\beta}\right)-T\left(a_{\alpha}\right)\right] \leq(1-\varepsilon)$. For every $n \in K$, there exist 
$\left(x_{n}, \alpha_{n}\right) \in \tilde{E}$ such that $\tilde{F}_{\tilde{A}}\left[\left(x_{n}, \alpha_{n}\right)-a_{\alpha}\right] \geq\left(1-\frac{1}{n}\right)$, but

$\tilde{F}_{\tilde{D}}\left[T\left(x_{n}, \alpha_{n}\right)-T\left(a_{\alpha}\right)\right] \leq(1-\varepsilon)$. The sequence $\left\{\left(x_{n}, \alpha_{n}\right)\right\}$ fuzzy converges to $a_{\alpha}$ but the sequence $\left\{T\left(x_{n}, \alpha_{n}\right)\right\}$ does not fuzzy converge to $\left(a_{\alpha}\right)$. This contradicts the assumption that every sequence $\left\{\left(x_{n}, \alpha_{n}\right)\right\}$ in $\tilde{E}$ fuzzy converging to $a_{\alpha}$ has the property that $T\left(x_{n}, \alpha_{n}\right) \rightarrow T\left(a_{\alpha}\right)$. Therefore the assumption that $T$ is not fuzzy continuous at $a_{\alpha}$ must be false.

\section{Definition 5.3:}

Let $\left(\tilde{A}, \tilde{F}_{\tilde{A}}, *\right)$ and $\left(\tilde{D}, \tilde{F}_{\tilde{D}}, *\right)$ be two fuzzy length spaces let $\tilde{E} \subseteq \tilde{A}$ and let $T: \tilde{E} \rightarrow \tilde{D}$ the fuzzy point $a_{\alpha}$ is a fuzzy limit of $\tilde{E}$ written by $\lim _{x_{\beta} \rightarrow a_{\alpha}} T\left(x_{\beta}\right)=b_{\beta}$ whenever $b_{\beta} \in \tilde{D}$ if for every $0<\varepsilon<1$ there is $0<\delta<1$ such that $\tilde{F}_{\tilde{D}}\left[T\left(x_{\beta}\right)-b_{\beta}\right]>(1-\varepsilon)$ when $x_{\beta} \in \tilde{E}$ and $\tilde{F}_{\tilde{A}}\left[x_{\beta}-a_{\alpha}\right]>(1-\delta)$.

\section{Proposition 5.4:}

Let $\left(\tilde{A}, \tilde{F}_{\tilde{A}}, *\right)$ and $\left(\tilde{D}, \tilde{F}_{\tilde{D}}, *\right)$ be two fuzzy length spaces, let $\tilde{E} \subseteq \tilde{A}$ and $T: \tilde{E} \rightarrow \tilde{D}$. Suppose that $a_{\alpha}$ is fuzzy limit fuzzy point of $\tilde{E}$. Then $\lim _{x_{\beta} \rightarrow a_{\alpha}} T\left(x_{\beta}\right)=b_{\beta}$ if and only if for every sequence $\left\{\left(x_{n}, \alpha_{n}\right)\right\}$ in $\tilde{E}$ such that $\left(x_{n}, \alpha_{n}\right) \neq a_{\alpha}$ and $\left(x_{n}, \alpha_{n}\right) \rightarrow a_{\alpha}$.

Proof: the argument is similar that of Theorem (5.3) and therefore is not included.

\section{Proposition 5.5:}

An operator $T$ of the fuzzy length space $\left(\tilde{A}, \tilde{F}_{\tilde{A}}, *\right)$ into a fuzzy length space $\left(\tilde{D}, \tilde{F}_{\tilde{D}}, *\right)$ is fuzzy continuous at the fuzzy point $a_{\alpha} \in \tilde{A}$ if and only if for every $0<\varepsilon<1$ there exist $0<\delta<1$ such that $\tilde{B}\left(a_{\alpha}, \delta\right) \subseteq T^{-1}\left[\tilde{B}\left(a_{\alpha}, \varepsilon\right)\right]$.

Proof: the operator $T: \tilde{A} \rightarrow \tilde{D}$ is fuzzy continuous at $a_{\alpha} \in \tilde{A}$ if and only if for every $0<\varepsilon<1$ there exist $0<\delta<1$ such that $\tilde{F}_{\tilde{D}}\left[T\left(x_{\beta}\right)-T\left(a_{\alpha}\right)\right]>(1-\varepsilon)$ for all $x_{\beta}$ satisfying $\tilde{F}_{\tilde{A}}\left[x_{\beta}-a_{\alpha}\right]>(1-\delta)$ i.e. $x_{\beta} \in \tilde{B}\left(a_{\alpha}, \delta\right)$ implies $T\left(x_{\beta}\right) \in \tilde{B}\left(T\left(a_{\alpha}\right), \varepsilon\right)$ or $T\left[\tilde{B}\left(a_{\alpha}, \delta\right)\right] \subseteq \tilde{B}\left(T\left(a_{\alpha}\right), \varepsilon\right)$ this is equivalent to the condition $\tilde{B}\left(a_{\alpha}, \delta\right) \subseteq T^{-1}\left[\tilde{B}\left(T\left(a_{\alpha}\right), \varepsilon\right)\right]$.

\section{Theorem 5.6:}

An operator $T: \tilde{A} \rightarrow \tilde{D}$ is fuzzy continuous if and only if $T^{-1}(\tilde{G})$ is fuzzy open in $\tilde{A}$ for all fuzzy open $\tilde{G}$ of $\tilde{D}$ where $\left(\tilde{A}, \tilde{F}_{\tilde{A}}, *\right)$ and $\left(\tilde{D}, \tilde{F}_{\tilde{D}}, *\right)$ are fuzzy length space.

\section{Proof:}

Suppose that $T$ is fuzzy continuous operator and let $\tilde{G}$ be fuzzy open in $\tilde{D}$ we show that $T^{-1}(\tilde{G})$ is fuzzy open in $\tilde{A}$. Since $\varnothing$ and $\tilde{A}$ are fuzzy open, we may suppose that $T^{-1}(\tilde{G}) \neq \varnothing$ and $T^{-1}(\tilde{G}) \neq \tilde{A}$.

Let $x_{\beta} \in T^{-1}(\tilde{G})$ then $T\left(x_{\beta}\right) \in \tilde{G}$. Since $\tilde{G}$ is fuzzy open, then there is $0<\varepsilon<1$ such that $\tilde{B}\left(T\left(x_{\beta}\right), \varepsilon\right) \subseteq \tilde{G}$, since $T$ is fuzzy continuous at $x_{\beta}$ by proposition 4.5 for this $\varepsilon$ there exist $0<\delta<1$ such that $\tilde{B}\left(x_{\beta}, \delta\right) T^{-1} \subseteq\left[\tilde{B}\left(T\left(x_{\beta}\right), \varepsilon\right)\right] \subseteq T^{-1}(\tilde{G})$.

Thus, every fuzzy point $x_{\beta}$ of $T^{-1}(\tilde{G})$ is an interior fuzzy point and so $T^{-1}(\tilde{G})$ if a fuzzy open in $\tilde{A}$.

Conversely, suppose that $T^{-1}(\tilde{G})$ is fuzzy open in $\tilde{A}$ for any fuzzy open $\tilde{G}$ of $\tilde{D}$. Let $x_{\alpha} \in \tilde{A}$ for each $0<\varepsilon<1$, the fuzzy ball $\tilde{B}\left(T\left(x_{\alpha}\right), \varepsilon\right)$ is fuzzy open in $\tilde{A}$ 
since $x_{\alpha} \in T^{-1}\left[\tilde{B}\left(T\left(x_{\alpha}\right), \varepsilon\right)\right]$ it follows that there exist $0<\delta<1$ such that $\tilde{B}\left(x_{\alpha}, \delta\right) \subseteq T^{-1}\left[\tilde{B}\left(T\left(x_{\alpha}\right), \varepsilon\right)\right]$ by proposition 5.5 , it follows that $T$ is fuzzy continuous.

\section{Theorem 5.7:}

An operator $T: \tilde{A} \rightarrow \tilde{D}$ is fuzzy continuous on $\tilde{A}$ if and only if $T^{-1}(\tilde{E})$ is fuzzy closed in $\tilde{A}$ for all fuzzy closed $\tilde{E}$ of $\tilde{D}$.

Proof:

Let $\tilde{E}$ be a fuzzy closed subset of $\tilde{D}$ then $\tilde{D}-\tilde{E}$ is fuzzy open in $\tilde{D}$ so that $T^{-1}[\tilde{D}-\tilde{E}]$ is fuzzy open in $\tilde{A}$ by theorem 4.6 but $T^{-1}[\tilde{D}-\tilde{E}]=\tilde{A}-T^{-1}(\tilde{E})$ so $T^{-1}(\tilde{E})$ is fuzzy closed in $\tilde{A}$.

Conversely, suppose that $T^{-1}(\tilde{E})$ is fuzzy closed in $\tilde{A}$ for all fuzzy closed subset $\tilde{E}$ of $\tilde{D}$. But the empty fuzzy set and the whole space $\tilde{A}$ are fuzzy closed fuzzy set. Then $\tilde{A}-T^{-1}(\tilde{E})$ is fuzzy open in $\tilde{A}$ and $T^{-1}[\tilde{D}-\tilde{E}]=\tilde{A}-T^{-1}(\tilde{E})$ is fuzzy in $\tilde{A}$. Since every fuzzy open subset of $\tilde{D}$ is of the type $\tilde{D}-\tilde{E}$, where $\tilde{E}$ is suitable fuzzy closed fuzzy set, it follows by using theorem 5.6, that $T$ is fuzzy continuous.

\section{Theorem 5.8:}

Let $\left(\tilde{A}, \tilde{F}_{\tilde{A}}, *\right)$ and $\left(\tilde{D}, \tilde{F}_{\tilde{D}}, *\right)$ and $\left(\tilde{Z}, \tilde{F}_{\tilde{Z}}, *\right)$ be three fuzzy length spaces let $T: \tilde{A} \rightarrow \tilde{D}$ and $S: \tilde{D} \rightarrow \tilde{Z}$ be fuzzy continuous operator. Then the composition SoT is a fuzzy continuous operator from $\tilde{A}$ into $\tilde{Z}$.

\section{Proof:}

Let $\tilde{G}$ be fuzzy open subset of $\tilde{Z}$. By theorem (5.6), $S^{-1}(\tilde{G})$ is a fuzzy open subset of $\tilde{D}$ and another application of the same theorem shows that $T^{-1}\left(S^{-1}(\tilde{G})\right)$ is a fuzzy open subset of $\tilde{A}$, since $(S o T)^{-1}(\tilde{G})=T^{-1}\left(S^{-1}(\tilde{G})\right)$ it follows from the same theorem again that ( $S o T$ ) is a fuzzy continuous.

\section{Theorem 5.9:}

Let $\left(\tilde{A}, \tilde{F}_{\tilde{A}}, *\right)$ and $\left(\tilde{D}, \tilde{F}_{\tilde{D}}, *\right)$ be two fuzzy length spaces let $T: \tilde{A} \rightarrow \tilde{D}$, then the following statements are equivalent.

(i) $T$ is fuzzy continuous on $\tilde{A}$.

(ii) $\overline{T^{-1}(\tilde{E})} \subseteq T^{-1}(\overline{\tilde{E}})$ For all subset $\tilde{E}$ of $\tilde{D}$.

(iii) $T^{-1}(\overline{\tilde{G}}) \subseteq \overline{T^{-1}(\tilde{G})}$ For all subset $\tilde{G}$ of $\tilde{A}$.

Proof: (i) $\Rightarrow$ (ii)

Let $\tilde{E}$ be a subset of $\tilde{D}$, since $\overline{\tilde{E}}$ is a fuzzy closed subset of $\tilde{D}, T^{-1}(\overline{\tilde{E}})$ is fuzzy closed in $\tilde{A}$. Moreover $T^{-1}(\tilde{E}) \subseteq T^{-1}(\overline{\tilde{E}})$ and so $\overline{T^{-1}(\tilde{E})} \subseteq T^{-1}(\overline{\tilde{E}})$.

[Recall that $\overline{T^{-1}(\tilde{E})}$ is the smallest fuzzy closed fuzzy set containing $T^{-1}(\tilde{E})$ ].

Proof: (ii) $\Rightarrow$ (iii)

Let $\tilde{G}$ be a subset of $\tilde{A}$, then if $\tilde{E}=T(\tilde{G})$, we have $\tilde{G} \subseteq T^{-1}(\tilde{E})$ and $\overline{\tilde{G}} \subseteq \overline{T^{-1}(\tilde{E})}$ thus $T(\overline{\tilde{G}}) \subseteq T\left(T^{-1}(\overline{\tilde{E}})\right)=\overline{\tilde{E}}=\overline{T(\tilde{G})}$.

Proof: (iii) $\Rightarrow$ (i)

Let $\tilde{S}$ be a fuzzy closed fuzzy set in $\tilde{D}$ and fuzzy set $T^{-1}(\tilde{S})=\tilde{S}_{1}$. By theorem 
(4.7), it is sufficient to show that $\tilde{S}_{1}$ is a fuzzy closed in $\tilde{A}$, that is, $\tilde{S}_{1}=\tilde{S}_{1}$ Now $T\left(\overline{\tilde{S}_{1}}\right) \subseteq \overline{T\left(T^{-1}(\tilde{S})\right)} \subseteq \overline{\tilde{S}}=\tilde{S}$ so that $\overline{\tilde{S}_{1}} \subseteq T^{-1}\left(T\left(\overline{\tilde{S}_{1}}\right)\right) \subseteq T^{-1}(\tilde{S})=\tilde{S}_{1}$.

\section{Conclusion}

In this research the fuzzy length space of fuzzy points was defined as a generalization of the definition of fuzzy norm on ordinary points. Based on the defined fuzzy length, most of the properties of the ordinary norm were proved.

\section{References}

[1] Zadeh, L. (1965) Fuzzy Sets. Information and Control, 8, 338-452. https:/doi.org/10.1016/S0019-9958(65)90241-X

[2] Bag, T. and Samanta, S. (2003) Finite Dimensional Fuzzy Linear Spaces. Fuzzy Mathematics, 11, 678-705.

[3] Bag, T. and Samanta, S. (2006) Fixed Point Theorems on Fuzzy Normed Spaces. Information Sciences, 176, 2910-2931. https:/doi.org/10.1016/j.ins.2005.07.013

[4] Wang, C.X. and Ming, M. (1993) Continuity and Boundness Mappings between Fuzzy Normed Spaces. Fuzzy Mathematics, 1, 13-24.

[5] Felbin, C. (1992) Finite Dimensional Fuzzy Normed Linear Spaces. Fuzzy Sets and Systems, 48, 239-248. https:/doi.org/10.1016/0165-0114(92)90338-5

[6] Golet, I. (2010) On Generalized Fuzzy Normed Spaces and Coincidence Theorems. Fuzzy Sets and Systems, 161, 1138-1144. https:/doi.org/10.1016/j.fss.2009.10.004

[7] Kider, J. (2012) New Fuzzy Normed Spaces. Baghdad Science Journal, 9, 559-564.

[8] Kider, J.R. (2013) Completeness of the Cartesian Product of Two Complete Fuzzy Normed Spaces. Engineering and Technology Journal, 31, 310-315.

[9] Kider, J.K. and Hussain, Z.A. (2014) Continuous and Uniform Continuous Mapping on Standard Fuzzy Metric Spaces. Engineering and Technology Journal, 32, 1111-1119.

[10] Cheng, S. and Mordeson, J. (1994) Fuzzy Linear Operators and Fuzzy Normed Linear Spaces. Bulletin of Calcutta Mathematical Society, 86, 429-436.

[11] Kramosil, O. and Michalek, J. (1975) Fuzzy Metrics and Statistical Metric Spaces. Kybernetika, 11, 326-334.

[12] George, A. and Veeramani, P. (1994) On Some Results in Fuzzy Metric Spaces. Fuzzy Sets and Systems, 64, 395-399. https:/doi.org/10.1016/0165-0114(94)90162-7 
Submit or recommend next manuscript to OALib Journal and we will provide best service for you:

- Publication frequency: Monthly

- 9 subject areas of science, technology and medicine

- Fair and rigorous peer-review system

- Fast publication process

- Article promotion in various social networking sites (LinkedIn, Facebook, Twitter, etc.)

- Maximum dissemination of your research work

Submit Your Paper Online: Click Here to Submit

Or Contact service@oalib.com 